\title{
PREVALENCE OF POSTEROLATERAL CORNER INJURIES IN MRI DETECTED ANTERIOR CRUCIATE LIGAMENT INJURIES
}

\author{
Sunil Parackal Ramakrishnan ${ }^{1}$, Saanida M. P2, V. R. Rajendran 3 , Noufal Perumpalath ${ }^{4}$, Jineesh Thottath 5 , Juvaina P6, Ipsit I. Menon ${ }^{7}$, \\ Sibin Surendran ${ }^{8}$
}

1 Junior Resident, Department of Radiodiagnosis, Government Medical College, Kozhikode.

${ }^{2}$ Assistant Professor, Department of Radiodiagnosis, Government Medical College, Kozhikode.

${ }^{3}$ Professor and HOD, Department of Radiodiagnosis, Government Medical College, Kozhikode.

${ }^{4}$ Associate Professor, Department of Radiodiagnosis, Government Medical College, Kozhikode.

${ }_{5}^{5}$ Assistant Professor, Department of Radiodiagnosis, Government Medical College, Kozhikode.

${ }^{6}$ Assistant Professor, Department of Radiodiagnosis, Government Medical College, Kozhikode.

${ }^{7}$ Radiologist, Department of Radiodiagnosis, Government Medical College, Kozhikode.

${ }^{8}$ Associate Professor, Department of Orthopaedics, Government Medical College, Kozhikode.

\section{ABSTRACT}

\section{BACKGROUND}

Anterior cruciate ligament injuries contribute to the major ligament injuries involving the knee. What we thought of isolated anterior cruciate ligament (ACL) injuries were not isolated injuries, there are associated posterolateral, posteromedial, anterolateral corner injuries and meniscal injuries. Identifying these injuries is of paramount importance to prevent the knee from developing arthritic changes in the future.

Objectives- To know the spectrum of posterolateral corner injuries in ACL injured knee.

\section{MATERIALS AND METHODS}

Patients who came to Radiology Department of Government Medical College, Kozhikode with positive history and symptoms of ACL injury, of which there was MRI evidence of ACL injury, were taken for studying the prevalence of posterolateral corner injuries. The study period was from January 2015 to September 2016.

Type of Study- Descriptive study.

Inclusion Criteria- Positive history of ACL lesion. Positive one or two ACL lesion physical tests (the Lachman, anterior drawer test and pivot test).

Exclusion Criteria- Patients $<18$ yrs. and $>80$ yrs. ACL injury with knee dislocation.

\section{RESULTS}

Posterolateral (PL) corner injuries showed prevalence of 5.1\% and all the cases were in grade 3 ACL injured knees. Isolated lateral collateral ligament showed prevalence of $10 \%$.

\section{CONCLUSION}

In cases of ACL injury undergoing MR imaging, posterolateral corner injuries have to be specifically looked and reported for better management of the patients.

\section{KEYWORDS}

Prevalence, ACL, Posterolateral Corner.

HOW TO CITE THIS ARTICLE: Ramakrishnan SP, Saanida MP, Rajendran VR, et al. Prevalence of posterolateral corner injuries in MRI detected anterior cruciate ligament injuries. J. Evolution Med. Dent. Sci. 2017;6(43):3380-3384, D0I: 10.14260/Jemds/2017/732

\section{BACKGROUND}

Knee injuries are one of the common problems in orthopaedics which include fractures and ligament injuries. Among the ligament injuries, anterior cruciate ligament (ACL) injuries account for the bulk of the injuries.

Now everyone recognises the importance that ACL is the primary restraint of anterior translation of tibia.

Financial or Other, Competing Interest: None.

Submission 19-04-2017, Peer Review 18-05-2017,

Acceptance 24-05-2017, Published 29-05-2017.

Corresponding Author:

Dr. Sunil Parackal Ramakrishnan,

Parackal House,

Opposite JFCM Court,

Court Road, Nilambur,

Malappuram-679329, Kerala.

E-mail: drsunilortho@gmail.com

DOI: $10.14260 /$ jemds $/ 2017 / 732$
Even now the debate continues whether to surgically repair the isolated ACL tear. Advocates of these groups say isolated tear of ACL alone can be managed by muscle strengthening exercise. The issues with regard to the isolated ACL injuries is that over the time there is increase in associated medial meniscus injury and osteochondral defects leading to secondary osteoarthritis. ${ }^{1-6}$ With advanced imaging and arthroscopic techniques, we came to know that what we thought of isolated ACL injuries were not isolated injuries.7 There are associated posterolateral, posteromedial or anterolateral corner injuries and meniscal injuries. Identifying these injuries is of paramount importance to prevent the knee developing arthritic changes in future.

Regarding the surgical management of ACL injury, present scenario is arthroscopic intra-articular reconstruction of the ligament with either hamstring tendon graft (Single bundle or quadruple bundle) or bone patellar bone tendon graft. 
Lateral corner of the knee can be divided into anterolateral and posterolateral corner. Posterolateral corner is stabilised by the arcuate ligament complex which consists of lateral collateral ligament; biceps femoris tendon; popliteus muscle and tendon; popliteal meniscal and popliteal fibular ligaments; arcuate ligament, lateral gastrocnemius muscle and fabellofibular ligaments. Most of the injuries involving the lateral corner injuries can be evaluated by MR imaging by routine spin echo sequences in the sagittal, coronal, and axial planes.

When comparing the injuries involving the medial and lateral structures of the knee, lateral quadrant injuries are less common than medial quadrant injuries; however, the lateral quadrant injuries are more disabling than medial quadrant injuries. ${ }^{8-10}$ And moreover lateral quadrant injuries are associated with injuries to the cruciates and injuries to the medial corner. ${ }^{11}$. These types of injuries are frequently missed in the clinical examination.

\section{MATERIALS AND METHODS}

Patients who came to Radiology Department of Government Medical College, Kozhikode with positive history and symptoms of ACL injury, of which there was MRI evidence of ACL injury, were taken for studying the prevalence of posterolateral corner injuries.

\section{Methodology \\ Research Design \\ Cross sectional study.}

\section{Duration of Study}

For a period of one and half years.

\section{Study Setting}

Department of Radiodiagnosis, Government Medical College, Calicut.

\section{Study Period}

Starting from January 2015 to September 2016.

\section{Study Subjects}

Patients above 18 and less than 80 years who had MR imaging evidence of ACL injuries were taken up for study.

\section{Inclusion Criteria}

- Positive history of ACL lesion.

- Positive one or two ACL lesion physical tests (the Lachman, anterior drawer test and pivot test).

\section{Exclusion Criteria}

- $\quad$ Patients $<18$ yrs. and $>80$ yrs.

- ACL injury with knee dislocation.

\section{Sample Size}

Sample size was calculated using the formula $\mathrm{n}=\mathrm{Z}^{2} \mathrm{pq} / \mathrm{d}^{2}$. $(\mathrm{Z}=1.96$, expected $\mathrm{p}=20$. (Prevalence was taken assuming that $20 \%$ of the patients are having positive anterior and posterolateral corner injuries)

$\mathrm{q}=100-\mathrm{p}=80$, precision $=7 \%$ ).

$\mathrm{N}=3.96 \times 20 \times 80 / 49=120$.

\section{Study Method}

Basic data and patient information was collected using proforma which include name, age and sex, involved side of knee, brief patient history and physical examination findings. Patients were selected for the study on the basis of injury involving the ACL and these injuries were graded into three grades; Grade 1: partial tear with less than half of the ligament substance disrupted; Grade2: partial tear with more than half of the ligament substance disrupted; Grade3: complete tear.

Injuries involving the posterolateral corner assessed according to the involvement of lateral collateral ligament, popliteus tendon, fabellofibular ligament, arcuate ligament by the hyperintense signals and bulkiness of the structures in T2 and PD weighted images, and discontinuity in the fibres in various grades of ACL injured knees.

\section{MRI Protocol}

MRI done with a 1.5 Tesla (GE HDXT) 16 channel with 18 superconducting shim coils and a dedicated 8 channel 13 element phased array knee coil with patient lying supine. The imaging protocols include mainly T1-weighted images in the axial plane (TR/TE, 400-700/9-16), T2-weighted images with fat saturation in the sagittal and coronal planes (TR/TE, 3200-4500/40-50), T2* -weighted images in sagittal (TR/TE 400-600/15-25) and FS PD-weighted images in axial, sagittal and coronal planes (TR/TE 3,000-4000/40-50) with slice thickness of $3 \mathrm{~mm}$ and spacing of $0.5 \mathrm{~mm}$.

\section{Statistical Analysis}

- Data was coded and entered in MS excel. Analysis was done using SPSS version 18.

- Frequencies of PL corner Injuries of knee were assessed as percentages.

- Association of PL corner injuries in various Grades of ACL assessed were expressed in percentages. Statistical test used was chi square test.

- P value $<0.05$ were taken as significant.

- Different statistical analytical methods were used to analyse the data.

\section{Variable under Study}

Spectrum of posterolateral corner injuries in various grades of ACL injured knees.

\section{Measurement of Variables}

Using T2 weighted sequences with fat saturation and proton density sequences.

\section{Ethical Aspects}

Study protocol was submitted to the institutional research committee as well as institutional ethics committee of Govt. Medical College, Kozhikode and clearance was obtained for conducting the study. 
Distribution of Posterolateral Corner Injuries in ACL Injured Knees

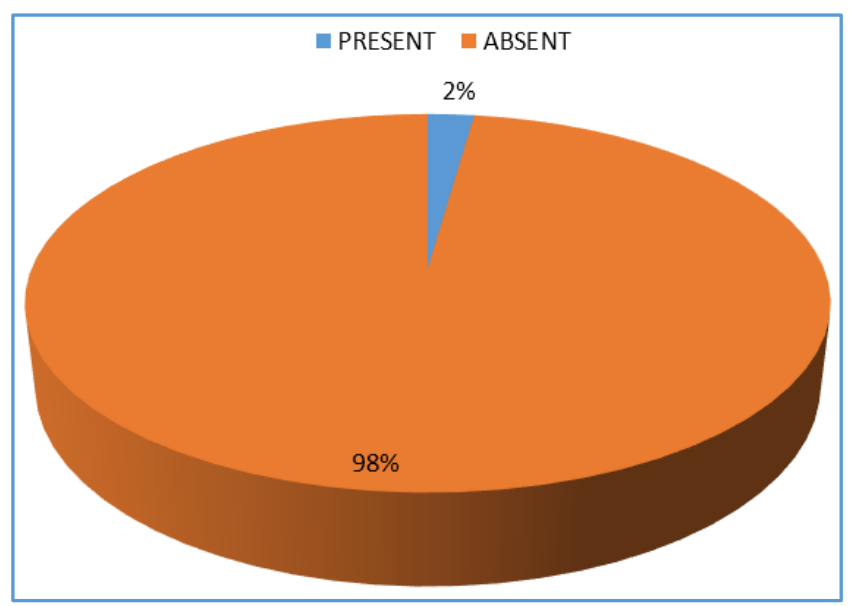

Figure 1. Distribution of Posterolateral Corner Injuries in ACL Injured Knees

Association of Posterolateral Corner Injuries in Various Grades of ACL Injured Knees

\begin{tabular}{|c|c|c|c|}
\hline Grades of & Posterolateral Corner Injuries & \multirow{2}{*}{ Total } \\
\cline { 2 - 3 } ACL Injuries & Present & Absent & \\
\hline Grade 1 ACL & 0 & 42 & 42 \\
injury & $.0 \%$ & $100.0 \%$ & $100.0 \%$ \\
\hline $\begin{array}{c}\text { Grade 2 ACL } \\
\text { injury }\end{array}$ & 0 & 59 & 59 \\
\hline Grade 3 ACL & 4 & $100.0 \%$ & $100.0 \%$ \\
injury & $5.1 \%$ & 75 & 79 \\
\hline Total & $\mathbf{4}$ & $94.9 \%$ & $100.0 \%$ \\
\hline \multicolumn{3}{|c|}{ Table 1 } \\
\hline
\end{tabular}

Chi square- 5.2, p-value-0.073.

Association of Isolated Lateral Collateral Ligament Tear in Various Grades of ACL Injured Knees

\begin{tabular}{|c|c|c|c|}
\hline Grades of & Lateral collateral ligament tear & \multirow{2}{*}{ Total } \\
\cline { 2 - 3 } ACL tear & Present & Absent & \\
\hline $\begin{array}{c}\text { Grade 1 ACL } \\
\text { injury }\end{array}$ & 3 & 39 & 42 \\
\hline $\begin{array}{c}\text { Grade 2 ACL } \\
\text { injury }\end{array}$ & $5.1 \%$ & $92.9 \%$ & $100.0 \%$ \\
\hline $\begin{array}{c}\text { Grade 3 ACL } \\
\text { injury }\end{array}$ & $8.5 \%$ & 54 & 59 \\
\hline Total & $10.1 \%$ & $81.5 \%$ & $100.0 \%$ \\
\hline \multicolumn{3}{|c|}{ Table 2 } & 79 \\
\hline
\end{tabular}

Chi square- 0.320 , p-value-0.852.
Case 1 showing injuries involving the popliteus tendon and popliteofibular ligament, the fabellofibular ligament, the biceps femoris tendon.

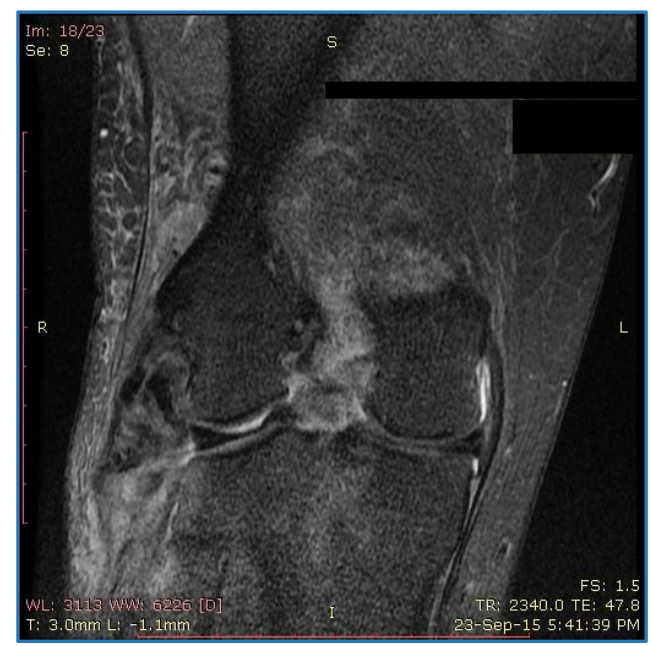

Figure A

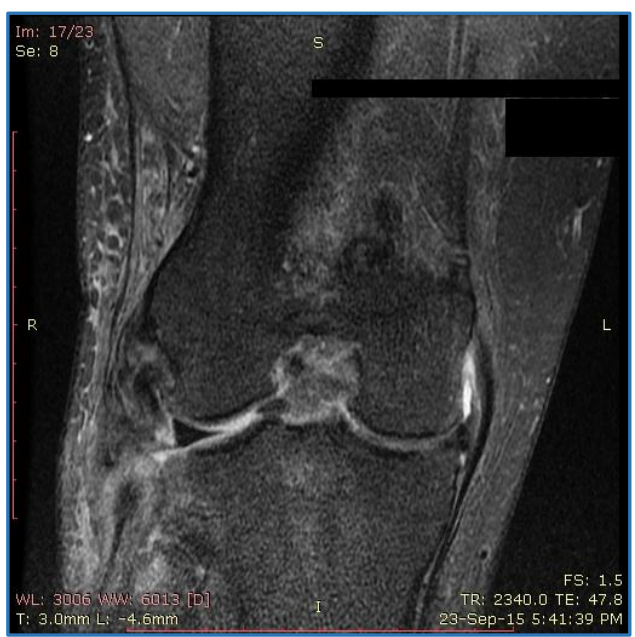

Figure B

Case 2

Showing injuries involving the posterolateral corner

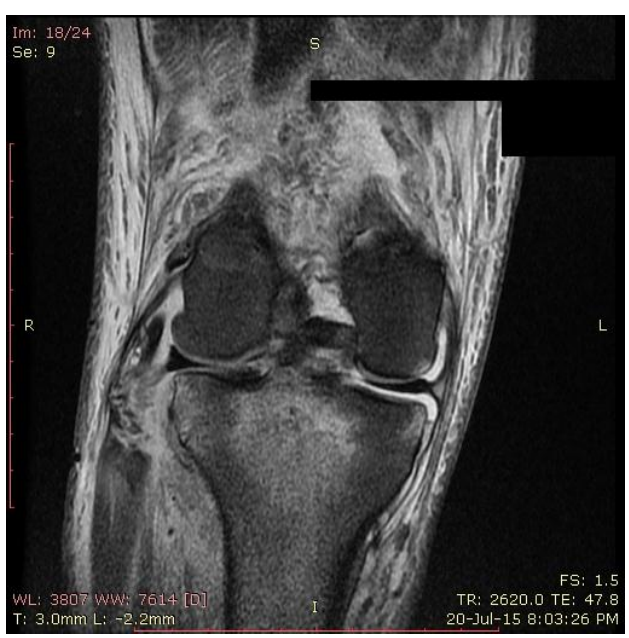

Figure A 


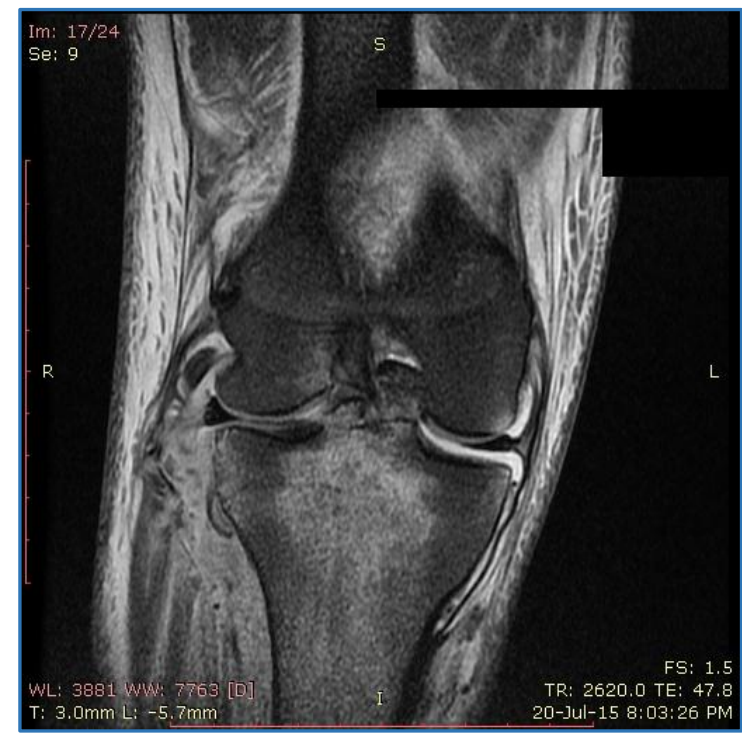

Figure B

\section{RESULTS}

We studied MRI of ACL injured knees for knowing the prevalence of posterolateral corner injuries in 180 patients who came to the Department of Radiology, Government Medical College, Calicut from January 2015 to September 2016.

In our study, majority of the patients with ACL injury were male (78\%) and showed equal involvement of right and left knees. A study by Csintalan ${ }^{12}$ showed higher incidence in male patients.

Among the various grades of ACL injured knees, 44\% cases showed grade 3 tear.

Posterolateral corner injuries were seen in 4 cases (5.1\%) with all the cases noted in Grade III ACL injuries. All these cases showed injury involving the popliteal tendon and lateral collateral ligament.

Injuries of the isolated lateral collateral ligament injuries were noted in $7.1 \%$ cases of grade 1 ACL injured knees and $8.5 \%$ and $10 \%$ cases of grade 2 and grade 3 ACL injured knees. A similar study by LaPrade RF et al ${ }^{13}$ showed $16 \%$ incidence of posterolateral injuries of knees when considering all ligament injuries. No separate incidence for each ACL and PCL injuries is mentioned in these articles.

An article by Bonadio $^{9}$ quotes the occurrence of posterolateral corner in $2 \%$ cases.

An article by Vinson et al quotes that with imaging alone it is difficult to define, when the instability of knee exists. With the available data, it can be observed that tear of two or more of the posterolateral structures (Especially LCL, popliteofibular ligament, posterolateral joint capsule) suggest the diagnosis of high grade PL corner injuries. ${ }^{14}$

According to this, our study showed only 4 cases with high grade PL corner injuries. Sixteen cases showed involvement of isolated LCL injuries in the various grades of ACL injured knees which was not stastically significant.

\section{CONCLUSION}

Majority of the anterior cruciate ligament (ACL) injured knees were noted in male patients and showed equal distribution in right and left knees.
All the PL (Posterolateral) corner injuries were noted in grade 3 ACL injured knees.

The knowledge about the anatomy and biomechanics of lateral corner injuries are researched and published.

In cases of ACL injury undergoing MRI, PL corner injuries have to be specifically looked and reported for better management of the patient.

\section{REFERENCES}

[1] Granan LP, Bahr R, Lie SA, et al. Timing of anterior cruciate ligament reconstructive surgery and risk of cartilage lesions and meniscal tears: a cohort study based on the Norwegian National Knee Ligament Registry. The American Journal of Sports Medicine 2009;37(5):955-61.

[2] Kennedy J, Jackson MP, O'Kelly P, et al. Timing of reconstruction of the anterior cruciate ligament in athletes and the incidence of secondary pathology within the knee. J Bone \& Joint Surg 2010;92(3):362-6.

[3] Lohmander LS, Englund PM, Dahl LL, et al. The longterm consequence of anterior cruciate ligament and meniscus injuries: osteoarthritis. The American Journal of Sports Medicine 2007;35(10):1756-69.

[4] Lohmander LS, Östenberg A, Englund M, et al. High prevalence of knee osteoarthritis, pain and functional limitations in female soccer players twelve years after anterior cruciate ligament injury. Arthritis \& Rheumatism 2004;50(10):3145-52.

[5] Mitsou A, Vallianatos P. Meniscal injuries associated with rupture of the anterior cruciate ligament: a retrospective study. Injury 1988;19(6):429-31.

[6] Tandogan RN, Taşer Ö, Kayaalp A, et al. Analysis of meniscal and chondral lesions accompanying anterior cruciate ligament tears: relationship with age, time from injury and level of sport. Knee Surgery Sports Traumatology Arthroscopy 2004;12(4):262-70.

[7] Schippinger G, Fellinger M, Passler J, et al. Management of the so called isolated ACL rupture. Acta Orthop Traumatol Turc 2004;25(5):260-2.

[8] Jakob RP, Hassler H, Staeubli HU. Observations on rotatory instability of the lateral compartment of the knee: experimental studies on the functional anatomy and the pathomechanism of the true and the reversed pivot shift sign. Acta Orthopaedica Scandinavica 1981;52(Suppl 191):1-34.

[9] Müller W, Biedert R, Hefti F, et al. OAK knee evaluation: a new way to assess knee ligament injuries. The knee and the cruciate ligaments. Heidelberg, Berlin: Springer 1992:123-33.

[10] Nicholas JA. Acute and chronic lateral instabilities of the knee: diagnosis, characteristics and treatment. In proceedings of the AAOS symposium on reconstructive surgery of the knee. Mosby 1978.

[11] Recondo JA, Salvador E, Villanúa JA, et al. Lateral stabilizing structures of the knee: functional anatomy and injuries assessed with MR imaging. Radiographics 2000;20 (Suppl 1):Spec No: S91-S102.

[12] Csintalan RP, Inacio MCS, Funahashi TT. Incidence rate of anterior cruciate ligament reconstructions. Perm J 2008;12(3):17-21. 
[13] LaPrade RF, Wentorf FA, Fritts $\mathrm{H}$, et al. A prospective magnetic resonance imaging study of the incidence of posterolateral and multiple ligament injuries in acute knee injuries presenting with a hemarthrosis. Arthroscopy: The Journal of Arthroscopic \& Related Surgery 2007;23(12):1341-7.
[14] Vinson EN, Major NM, Helms CA. The posterolateral corner of the knee. American Journal of Roentgenology 2008;190(2):449-58. 